\title{
A RARE FORM OF PRESENTATION OF A SMALL INTESTINE GIST IN THE EMERGENCY DEPARTMENT
}

Dan Nicolae Păduraru ${ }^{1,2}$, Ion Daniel ${ }^{1,2}$, Alexandra Bolocan ${ }^{1,2}$, Florentina Mușat ${ }^{2}$, Cosmin Alexandru Palcău ${ }^{2}$, Andrada Artamonov ${ }^{1}$, Octavian Andronic ${ }^{1,2}$

${ }^{1}$ Carol Davila University of Medicine and Pharmacy, Bucharest, Romania

${ }^{2}$ University Emergency Hospital of Bucharest, Romania

\section{CASE REPORT Abstract}

Doi: $10.33695 /$ rojes.v2i2.31 Accepted: 23.11.2020
Gastro-intestinal stromal tumors (GIST) are a form of mesenchymal tumors most commonly located in the gastro-intestinal tract, as the name suggests. The symptoms that might occur can be abdominal pain or discomfort, early satiety, bloating, jaundice, fatigue, hematemesis or melena. Most of GISTs (>95\%) are positive for the KIT protein. Surgery remains the only curative treatment for GIST amenable for resection. High risk tumors can receive adjuvant or neoadjuvant treatment with Imatinib Mesylate, a tyrosin-kinase inhibitor. We present the case of a 59-years old patient that presented with rectal bleeding and abdominal diffuse pain. Blood tests shows leukocytosis and moderate anemia. CT scan describes an expansive, polycyclic process projected at the limit between the mesogastric region and the left flank, in close contact with the small intestine without any clear demarcation line between them and at the superior pole of the tumor, located in an enteral loop, a band of hyperdensity with net contour. Intraoperative, an extraluminal jejunal tumor was found, located at 15 $\mathrm{cm}$ from the duodeno-jejunal flexure, with intraluminal active bleeding, which lead us to suspect a GIST based on the macroscopic aspect. We performed segmental enterectomy of $10 \mathrm{~cm}$ of the small intestine containing the tumor with entero-enteral anastomosis end to end. The histopathological and immunohistochemistry examinations correlated with the macroscopic aspect have confirmed the diagnosis of GIST.

Corresponding author:

Bolocan Alexandra bolocan.alexa@gmail.com
Keywords: GIST, gastro-intestinal stromal tumor, complicated GIST, hemorrhage

\section{Introduction}

Gastro-intestinal stromal tumor (GIST) is a mesenchymal tumor that was relatively recent discovered, usually being confused in the past with leiomyoma, leiomyosarcoma or leiomyoblastoma. From a morphological point of view GISTs could be epithelioid, spindlecell or pleomorphic tumors. With the help of the evolution of the modern day immunohistochemistry and electronic microscopy, we can now say with certainty 
that GISTs have a completely different kind of physiopathology: the activating mutation of the c-KIT tyrosine-kinase [1-3].

The main presentation is in the stomach (up to $70 \%$ ) and small intestine (20-30\%), but they can also occur in the esophagus, colon or rectum. Other locations like the omentum, mesentery or retroperitoneum are extremely rare $[4,5]$.

GISTs has an extremely different potential of malignancy that could vary from small, benign tumors to very aggressive sarcomas.

The symptoms could vary depending on the location, the size of the tumor, so the patients could encounter abdominal pain or discomfort, early satiety, bloating, jaundice, fatigue, palpitation, hematemesis, melena [6]. The patients may also present with an abdominal mass and no other symptoms or, as other studies suggest, 10 to $25 \%$ of the patients present with metastases [7]. As some studies had shown, massive hemorrhage is rarely the first or main symptom that a patient can experience $[8,9]$.

Classical therapeutic management is the surgical en-bloc removal of the tumor or, in case of metastatic disease, Imatinib Mesylate treatment prior to surgery. Depending on the location and size the removal of the GIST may require gastrectomy, pancreaticoduodenectomy or an abdominoperineal resection [3].

\section{Case presentation}

A 59-year old patient came to the Emergency Department with recurrent episodes of rectal bleeding and abdominal diffuse pain, symptomatology which started 7day prior his admission and escalated quickly in the last 12 hours. The patient was known with cardiac failure NYHA II, high blood pressure, acute hemorrhagic gastritis, esophagitis caused by gastro-esophageal reflux disease, hepatic steatosis and cystic tumor in the liver's second segment (confirmed by CT scan).

Clinical exam reveals pale complexion, diaphoresis and diffuse abdominal pain with signs of generalized abdominal guarding. Rectal examination indicates large quantity of melaena.

Initial blood tests revealed leukocytosis $\left(31.23 \mathrm{~m} / \mathrm{mm}^{3}\right)$ without any other sign of infection and moderate anemia $(\mathrm{Hb}=8,1$ $\mathrm{mg} / \mathrm{dL})$.

An abdominal and pelvic native and contrast CT was mandatory. In our patient's case, this investigation showed an expansive, polycyclic process $(67 / 49 / 81 \mathrm{~mm})$ projected at the limit between the mesogastric region and the left flank, non-homogenous post contrast, with hypodense areas, in close contact with the small intestine without any clear demarcation line between them (Figure 1). At the superior pole of the tumor, located in an enteral ansae, a band of hyperdensity (39/9/3 mm) with net contour- most likely a foreign object (Figure $3)$. Furthermore, the CT scan reveals an adenopathic mass $(26 / 10 / 46 \mathrm{~mm})$ situated anterior to the inferior vena cava, adjacent to the caudate lobe and multiple adenopathies located in close contact to the small gastric curvature.

The patient was scheduled for emergency surgery under general anesthesia. Intraoperative, an extraluminal jejunal tumor with a diameter of $10 \mathrm{~cm}$ was found, located at $15 \mathrm{~cm}$ from the duodeno-jejunal flexure, with rich vascularisation, invasive in the serosa, with intraluminal active bleeding (Figure 4,5) which lead us to suspect a gastro-intestinal stromal tumor based on the macroscopic aspect. No other organs were affected. We performed segmental enterectomy of approximately $10 \mathrm{~cm}$ of the small intestine containing the tumor with entero-enteral anastomosis end to end (Figures 6,7).

The resected segment was sent for histopathological examination. The result describes the $7 \mathrm{~cm}$ tumor as a gastro-intestinal stromal tumor with fusiform pattern with a 
mitotic index of $<5$ mitosis / $5 \mathrm{~mm}^{2}$, completely excised.

The immunohistochemistry examination shows positive results for CD117, DOG1, CD34, ACT and ki67. The histopathological and immunohistochemistry examinations correlated with the macroscopic aspect have confirmed the diagnosis of GIST, prognosis group 3a, medium risk of tumor progression $24 \%$ AFIP.

The patient's evolution was favorable and the clinical symptoms disappeared, remaining stable in the postoperative period and was discharged home in stable condition.

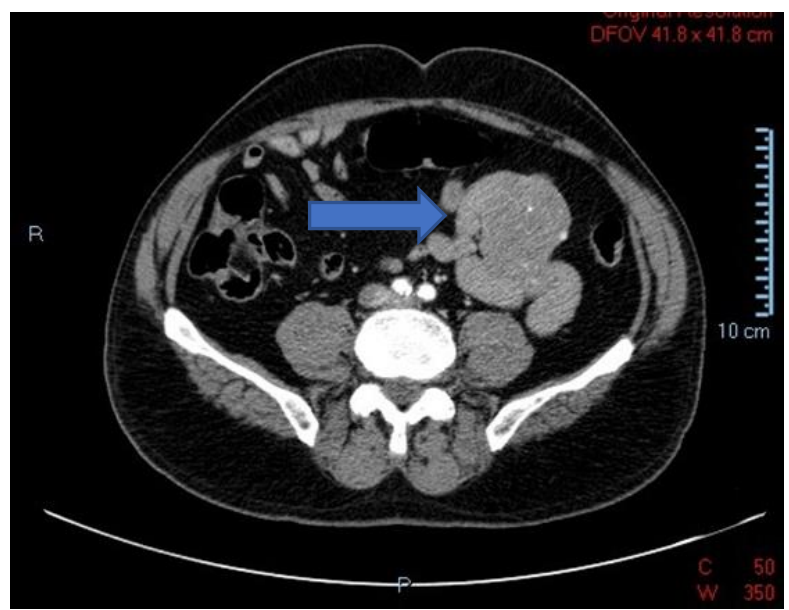

Figure 1 - Abdominal CT scan- transversal plain - The arrow points to the expansive, polycyclic process which is in close contact with the small intestine

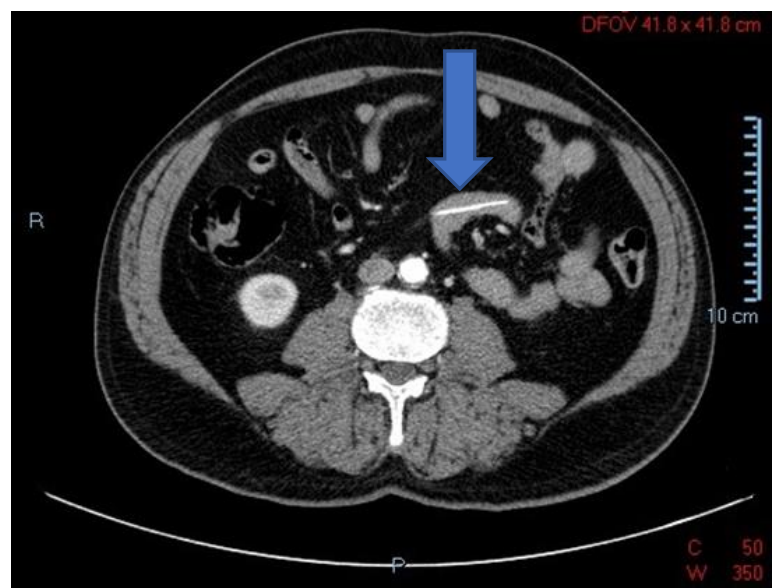

Figure 2 - Abdominal CT scan- transversal plain - The arrow points to the foreign object

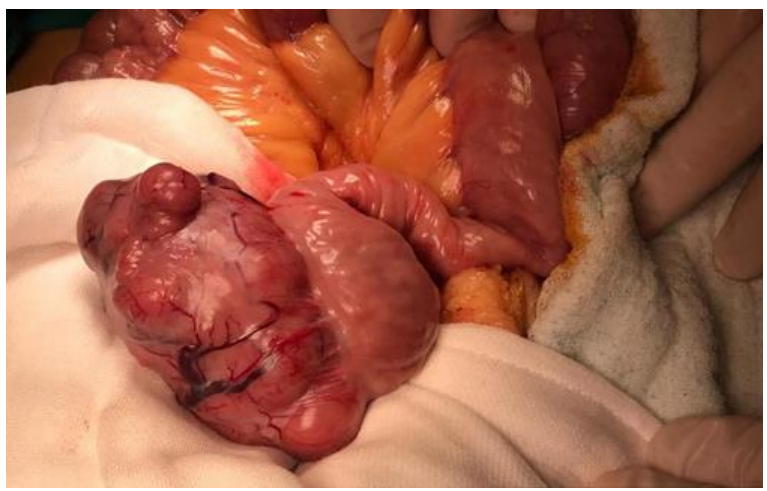

Figure 3 - Intraoperatory aspect showing the intestinal tumor located at $15 \mathrm{~cm}$ from the duodeno-jejunal flexure with rich vascularisation

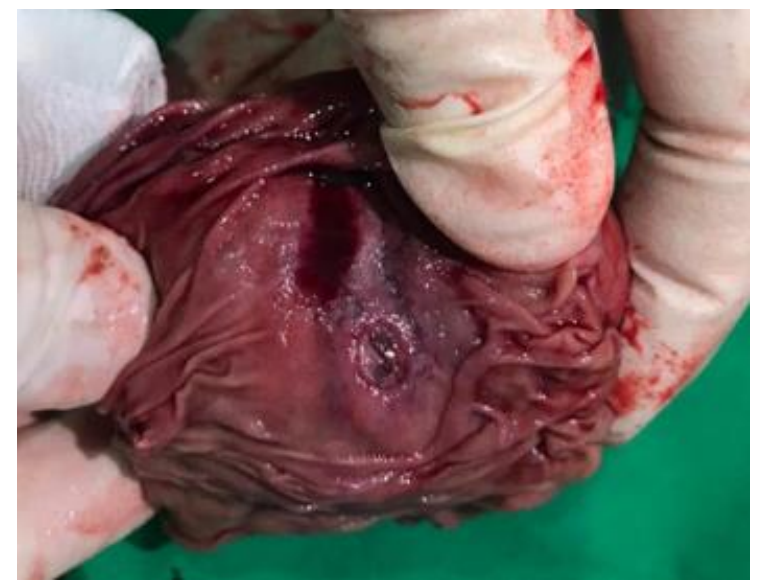

Figure 4 - Resection piece showing the ulcerated mucosae

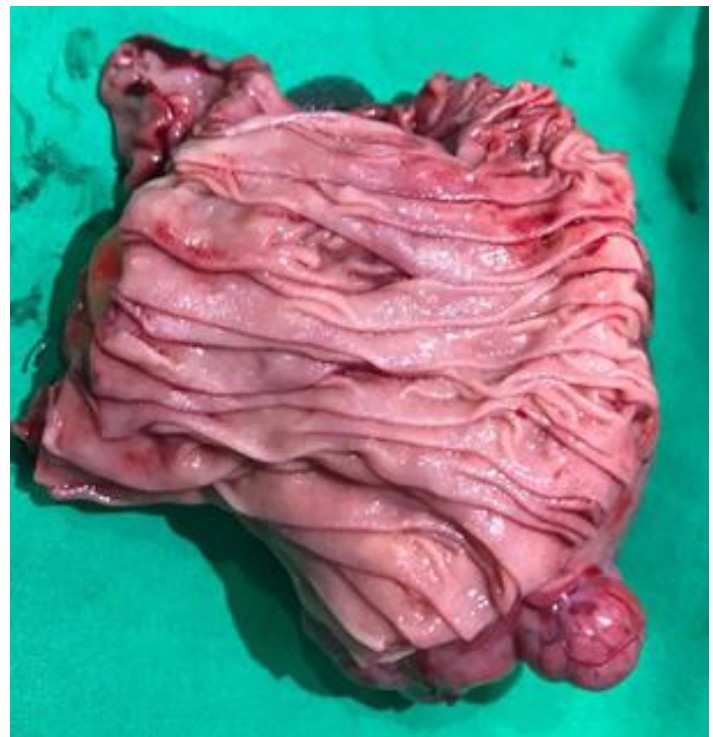

Figure 5 - Resection piece showing the intestinal mucosae 


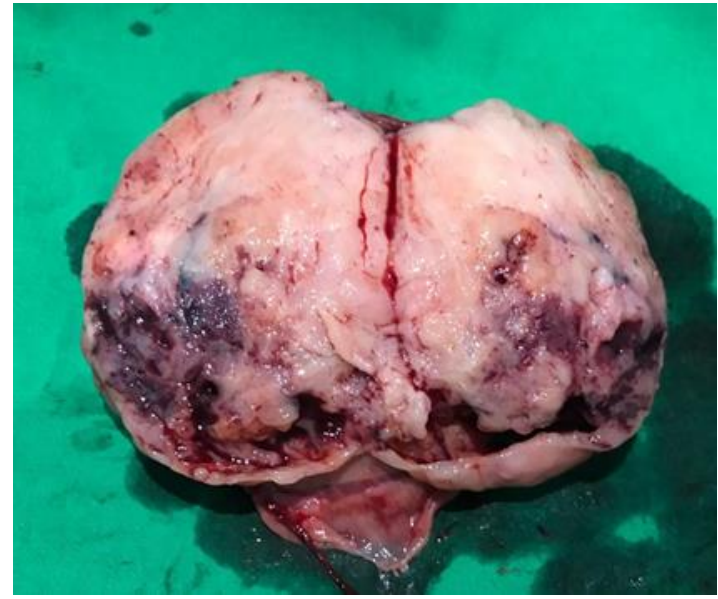

Figure 6 - Sectioned tumor showing the macroscopic aspect of the tumor

\section{Discussions}

Gastro-intestinal stromal tumors are a rare pathology, constituting less than $1 \%$ of all gastro-intestinal tract tumors [10]. Studies are showing an incidence varying from 4.3 per million [11] to 21.1 per million cases [12]. The clinical presentation of primary GIST's is split into two categories: symptomatic $(81,3 \%)$ and incidental $(18,7 \%)$ [13]. The patients are usually asymptomatic for long periods of time, GISTs being usually incidentally discovered in routine investigations. Among the most common symptoms were noted abdominal pain, gastro-intestinal obstruction and bleeding. However, life-threatening hemorrhage as the only symptom of the presence of the tumor in the small intestine is a rare scenario in which case the surgery becomes mandatory, as it did in our case.

This kind of presentation is rare, acute, massive bleeding from a GIST being rarely the cause of presentation in the emergency department, patients being usually diagnosed incidentally or they can suffer of other chronic symptoms that lead them to investigations [14]. Other form of GIST's emergency presentation is perforation. This situation also leads to emergency surgery for proper diagnostic and treatment [15].
Gastro-intestinal stromal tumors are known for their unspecified clinical presentation. They can be easily misdiagnosed as leiomyoma, leiomyosarcoma, neuroendocrine tumors, inflammatory polyps and many other benign or malign lesions [16]. They can also be confused with abscesses as other studies have shown [17].

In our particular case the diagnostic suspicion dictated by the CT scan and blood tests was foreign object inserted in the intestinal wall causing an abscess. The characteristic features of gastro-intestinal stromal tumors described on the CT images may frequently lead to diagnostic errors, as other studies have shown [18]. In this situation the correct approach for diagnostic and treatment is exploratory laparotomy and resection of the tumor, as the Report of GIST Consensus Conference shows [19].

$\mathrm{CT}$ is very useful in the diagnosis of the tumor, but the determination of the malignancy in GIST's is difficult and cannot be resolved with use of imaging and histopathological features alone. The results of immunohistochemistry positive for CD117, DOG1, CD 34 are the correct choice in the diagnosis of gastro-intestinal stromal tumor [20].

The treatment for a severe bleeding GIST is emergency surgery, but the prognosis after resection is influenced by completeness of the excision (R0) and the malignant potential of such tumor. Adjuvant treatment may be needed for patients with incomplete resection or high grade GIST (Imatinib Mesylate). Complete surgical resection is considered sufficient for low grade GIST [21].

\section{Conclusion}

Even though gastro-intestinal stromal tumors are rare, they should always be considered in the differential diagnoses of hemorrhagic gastro-intestinal masses. 


\section{References}

[1] G. Tryggvason, H. G. Gíslason, M. K. Magnússon, and J. G. Jónasson, "Gastrointestinal stromal tumors in Iceland, 1990-2003: the icelandic GIST study, a population-based incidence and pathologic risk stratification study.," Int. J. cancer, vol. 117, no. 2, pp. 289-293, Nov. 2005.

[2] E. Fülöp, S. Marcu, D. Milutin, and A. Borda, "Gastrointestinal stromal tumors: review on morphology, diagnosis and management.," Rom. J. Morphol. Embryol. = Rev. Roum. Morphol. Embryol., vol. 50, no. 3, pp. 319-326, 2009.

[3] H. Joensuu, "Gastrointestinal stromal tumor (GIST)," Ann. Oncol., vol. 17, no. SUPPL. 10, pp. x280-x286, 2006.

[4] C. Mucciarini et al., "Incidence and clinicopathologic features of gastrointestinal stromal tumors. A population-based study," BMC Cancer, vol. 7, no. 1, p. 230, 2007.

[5] C. L. Corless, J. A. Fletcher, and M. C. Heinrich, "Biology of gastrointestinal stromal tumors," J. Clin. Oncol., vol. 22, no. 18, pp. 3813-3825, 2004.

[6] M. Miettinen, L. H. Sobin, and J. Lasota, "Gastrointestinal stromal tumors of the stomach: a clinicopathologic, immunohistochemical, and molecular genetic study of 1765 cases with long-term followup.," Am. J. Surg. Pathol., vol. 29, no. 1, pp. 52-68, Jan. 2005.

[7] T. Tran, J. A. Davila, and H. B. El-Serag, "The epidemiology of malignant gastrointestinal stromal tumors: an analysis of 1,458 cases from 1992 to 2000.," Am. J. Gastroenterol., vol. 100, no. 1, pp. 162-168, Jan. 2005.

[8] M. Ajduk et al., "Spontaneously ruptured gastrointestinal stromal tumor (GIST) of the jejunum mimicking acute appendicitis.," Coll. Antropol., vol. 28, no. 2, pp. 937-941, Dec. 2004.

[9] S. Lahoti and N. Fukami, "The small bowel as a source of gastrointestinal blood loss.," Curr. Gastroenterol. Rep., vol. 1, no. 5, pp. 424-430, Oct. 1999.

[10] T. Acherjee et al., Gastrointestinal Stromal Tumor (GIST): A Rare Cause of Hematemesis and Hematochezia in an Otherwise Healthy 39-Year-Old Female. no. Md, pp. 3465-3466, 2020.

[11] Z. Wang, X. Liang, Y. Wang, G. Ma, Y. $\mathrm{Qu}$, and $\mathrm{X}$. Tian, "[Epidemiology survey of gastrointestinal stromal tumor in Shanxi Province in 2011]," Zhonghua Yi Xue Za Zhi, vol. 93, no. 32, pp. 2541-2544, 2013, [Online]. Available:

http://europepmc.org/abstract/MED/2435159 2.

[12] M. Lv, C. Wu, Y. Zheng, and N. Zhao, "Incidence and Survival Analysis of Gastrointestinal Stromal Tumors in Shanghai: A Population-Based Study from 2001 to 2010," Gastroenterol. Res. Pract., vol. 2014, p. 834136, 2014.

[13] K. Søreide, O. M. Sandvik, J. A. Søreide, V. Giljaca, A. Jureckova, and V. R. Bulusu, "Global epidemiology of gastrointestinal stromal tumours (GIST): A systematic review of population-based cohort studies," Cancer Epidemiol., vol. 40, pp. 39-46, 2016.

[14] S. Khuri, H. Gilshtein, A. Darawshy, H. Bahouth, and Y. Kluger, "Case Report Primary Small Bowel GIST Presenting as a LifeThreatening Emergency: A Report of Two Cases," vol. 2017, pp. 5-9, 2017.

[15] J. R. A. Skipworth, A. E. E. Fanshawe, and M. J. West, "Perforation as a rare presentation of gastric gastrointestinal stromal tumours: a case report and review of the literature," pp. 1-5, 2014.

[16] H. H. Wong et al., "Mimics of gastrointestinal stromal tumors (GISTs): Implications for diagnosis and managementThe Cambridge GIST Study Group (CGSG) experience.," J. Clin. Oncol., vol. 31, no. 15_suppl, pp. e21503-e21503, May 2013. [17] Y. Chen, Y. Han, and J. Du, "Diagnostic challenges in differentiating intramural gastric abscess from gastric cancer: Two case 
reports," Med. (United States), vol. 97, no. 43, pp. 2-5, 2018.

[18] A. Werewka-Maczuga, T. Osiński, R. Chrzan, M. Buczek, and A. Urbanik, "Characteristics of computed tomography imaging of gastrointestinal stromal tumor (GIST) and related diagnostic problems," Polish J. Radiol., vol. 76, no. 3, pp. 38-48, 2011.

[19] J. Blay et al., "Consensus meeting for the management of gastrointestinal stromal tumors Report of the GIST Consensus
Conference of 20 - 21 March 2004 , under the auspices of ESMO," Ann. Oncol., vol. 16, no. 4, pp. 566-578, 2005.

[20] M. Novelli et al., "DOG1 and CD117 are the antibodies of choice in the diagnosis of gastrointestinal stromal tumours," Histopathology, vol. 57, no. 2, pp. 259-270, 2010.

[21] P. Bucher et al., "An audit of surgical management of gastrointestinal stromal tumours (GIST)," Eur. J. Surg. Oncol., vol. 32, no. 3, pp. 310-314, 2006. 